\title{
Contingent and noncontingent response rates as a function of delay of reinforcement
}

\author{
MICHAEL P. SILVER and CHARLES H. PIERCE, DePauw \\ University, Greencastle, Ind. 46135
}

Rats were exposed to a VI 1-min reinforcement schedule in which reinforcement was delayed for varying intervals, and responses during the delay periods had no programmed effects. Response rates during the schedule declined as the delay length increased, but rates during the delay periods were not a simple function of delay length.

Previous free-operant studies of delay of reinforcement have demonstrated that responding preceding the delay, termed "contingent," decreases as the delay increases (Skinner, 1938, pp. 139, ff; Ferster, 1953; Dews, 1960). However, some studies (Ferster, 1953; Azzi, Fix, Keller, \& Rocha e Silva, 1964; Ferster \& Hammer, 1965) have shown that with cued delays, contingent rates can be maintained at relatively long delay intervals by means of special training procedures. In general, all these studies have minimized or eliminated responding during the delay, termed "noncontingent," by having every delay response reset the interval clock, or by use of a blackout. In the present experiment, contingent rates were studied as a function of several cued delay intervals under a procedure in which responses during the intervals had no programmed effects. This allowed comparision of both contingent and noncontingent rates at several values of delay interval.

\section{METHOD}

The Ss were six naive male albino rats of the Wistar strain, maintained at approximately $80 \%$ of their free-feeding weight throughout the experiment.

The apparatus consisted of two Scientific Prototype Model A-100 test cages enclosed in sound-resistant outer shells, and supplied with a continuous "white" masking noise. Each test cage had a lever (Scientific Prototype PLS-200) with a cue light mounted above it, and a food pellet dispenser. Automatic programming apparatus was located in an adjacent room.

For all Ss, bar-pressing was first reinforced with $10 \mathrm{sec}$ of light, and terminated by a food pellet. Conditioning occurred for all Ss within three 1-h sessions, during which time virtually no responses were made in the presence of the light. The Ss were then placed on a variable interval schedule with a mean of $1 \mathrm{~min}$ (VI $1 \mathrm{~min}$ ), with the reinforcement still following $10 \mathrm{sec}$ of light. After the contingent response rates (i.e., rates occurring in the absence of the light) had stabilized, the Ss were divided into two groups of three Ss each. Maintaining the basic VI 1-min schedule which preceded the delay, each group was exposed to a series of delay intervals, remaining at each interval for 20 daily 4 h sessions. For one group, the values of the delay and order of presentation were $10,20,40,80$, and $160 \mathrm{sec}$. For the other group this order was reversed.

Each animal was returned to its initial delay value (i.e., whether 10 or $160 \mathrm{sec}$ ) for 20 additional sessions after the sequence had been completed, as a measure of reversibility.

\section{RESULTS AND DISCUSSION}

Contingent and noncontingent rates at each delay value are presented in Figs. 1 and 2, respectively. In general, contingent rates decline as the delay increases, thus supporting a general finding in the literature. Noncontingent rates, however, do not seem to be a simple function of the delay length. Some Ss' rates (e.g., Rats 2 and 3) decline as the delay increases, while others (e.g., Rats 1 and 4) show increases.

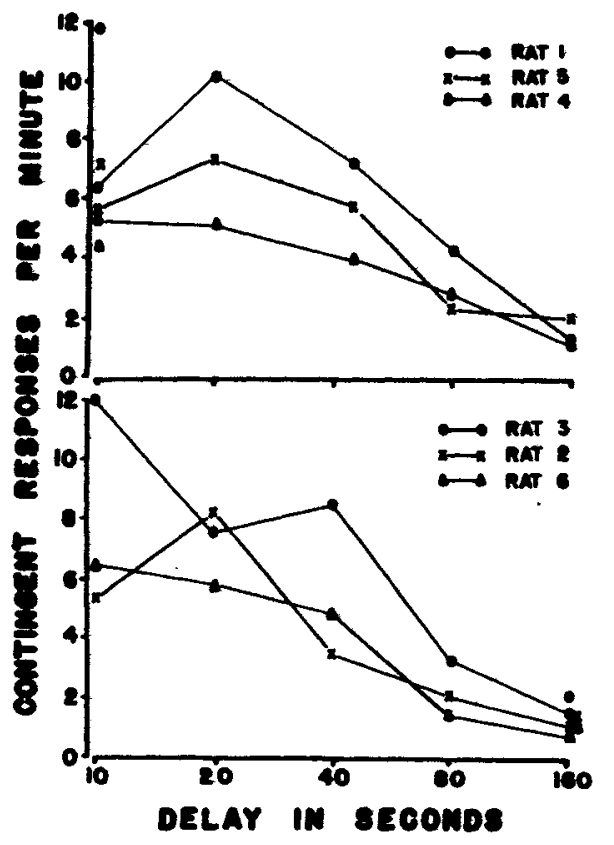

Fig. 1. Contingent response rates as a function of delay in seconds. Ss in upper graph received short to long delay sequence; Ss in lower graph received the reverse. Unconnected data points are recovery data.

Neither the contingent nor the noncontingent data show any consistent differences between the two groups due to the order of sequencing delay intervals. Data from the final procedure of returning each animal to its initial condition further indicate that sequences were not important. These data are shown on Figs. 1 and 2 as unconnected data points.

The ratio of noncontingent to contingent rates provides a comparison of the two types of responses. When no responses occur during the delay interval the ratio is zero. When noncontingent responding is equal to or greater than contingent responding, the value is 1.0 or more. These ratios for each animal at each delay interval are presented in Table 1. A great deal of variability obviously exists among the Ss. Although, as previously noted, responding was initially nonexistent during the delay, some Ss, particularly Rat 6, displayed high noncontingent rates later in the experiment.

Since the constant delay preceding reinforcement might indicate the reinforcement schedule to be functionally a chained VI-FI schedule, the Es collected further data on the noncontingent responses during the last two sessions at each

Table 1

Noncontingent Rate/Contingent Rate Ratios for Each $S$ at Each Delay Vahe

\begin{tabular}{llccccc}
\hline & & \multicolumn{5}{c}{ Delay } \\
& & $10 \mathrm{sec}$ & $20 \mathrm{sec}$ & $40 \mathrm{sec}$ & $80 \mathrm{sec}$ & $160 \mathrm{sec}$ \\
\cline { 3 - 7 } RAT & 1 & 0.33 & 1.52 & 2.51 & 4.12 & 4.13 \\
RAT & 2 & 0.22 & 0.34 & 0.04 & 0.06 & 0.13 \\
RAT & 3 & 0.19 & 0.13 & 0.07 & 0.06 & 0.20 \\
RAT & 4 & 0.65 & 0.07 & 0.04 & 0.83 & 5.56 \\
RAT & 5 & 0.46 & 0.30 & 0.20 & 0.46 & 0.27 \\
RAT & 6 & 0.64 & 0.65 & 1.09 & 2.08 & 3.00 \\
\hline
\end{tabular}






Fig. 2. Noncontingent response rates as a function of delay in seconds. Ss in upper graph received short to long delay sequence; $S s$ in lower graph received the reverse. Unconnected data points are recovery data. delay value. Numbers of responses were recorded in each of several subintervals of the delay value. Such an analysis would reveal particular patterns of responding, such as a fixedinterval "scallop." These data showed no systematic trends, either within or between Ss, and hence are not shown.

The lack of order in the noncontingent data might be predicted from Ferster's (1953) analysis of light acting as both a conditioned reinforcer for contingent responding, and as an $S^{D}$ for other behaviors which might adventitiously be reinforced by pellet delivery. On the other hand, this analysis would not be supported by the subinterval data if it were predicted that lever pressing, a prepotent response, would show consistency during the delay interval. Therefore, the data presented here cannot, at the present time, be viewed as supporting any particular interpretation.

\section{REFERENCES}

AZZI, R., FIX, D. S. R., KELLER, F. S., \& ROCHA E SILVA, M. I. Extereoceptive control of response under delayed reinforcement. Joumal of the Experimental Analysis of Behavior, 1964, 7, 159-162.

DEWS, P. B. Free-operant behavior under conditions of delayed reinforcement. I. CRF-type schedules. Journal of the Experimental Analysis of Behavior, 1960, 3, 221-234.

FERSTER, C. B. Sustained behavior under delayed reinforcement. Journal of Experimental Psychology, 1953, 45, 218-224.

FERSTER, C. B., \& HAMMER, C. Variables determining the effects of delay in reinforcement. Journal of the Experimental Analysis of Behavior, 1965, 8, 243-254.

SKINNER, B. F. The behavior of organisms. New York: Appleton Century Company, 1938.

(Continued from page 230)

and FR 4, respectively. The Ss given simple extinction without punishment averaged 234 responses, the same number as FR 1 satiation Ss.

Punishment, on either PR 1 or PR 4 schedules, was highly effective in suppressing responding at all three reward ratios. No PR 1 rat made more than seven responses or received more than five pellets; no PR 4 animal made more than 24 responses or received more than 24 pellets. The PR 16 animals showed much less suppression. They made from 32 to 373 responses; seven of the 12 Ss made more than 100 responses. Pellets received ranged from 8 to 241 , although only two Ss earned over 100 pellets. Thus, there was at least some overlap of PR 16 performance with that of nonpunished Ss. At the most favorable reward ratio, the PR 16 rats averaged $42 \%$ of the pellets received by satiation Ss. This implies that at a higher punishment ratio, perhaps around $P R 40$, satiation would occur before suppression.

In one sense it is a foregone conclusion that Ss punished less frequently will make more responses. For example, a PR 16 animal who quits responding after three shocks will have made at least 48 responses while a PR 1 rat who also stopped responding after three shocks would only have three responses to his credit. However, the pertinent fact is that the PR $16 \mathrm{Ss}$ took more shocks than either PR 1 or PR 4 Ss. If one looks at the distribution of shocks received in the PR 1 and PR 4 conditions, the number of shocks range from one to seven with a mode (and median) of three. Six of the 12 PR 16 Ss took more than seven shocks.

Punishment was also applied at three different ratios when no reward was given. The extinction-with-punishment animals quit responding after a few shocks, even with the PR 16 condition.

\section{DISCUSSION}

Theoretically, the effects of punishment should depend upon both punishment parameters (e.g., punishment intensity) and factors related to the strength of the behavior being punished (e.g., reward magnitude). However, reward variables often count for little in punishment studies. In the present experiment punishment quickly stopped responding under all conditions save those involving reward coupled with infrequent punishment. The sustaining function of reward was only revealed from the effect of its absence in the case of punished extinction. When reward is discontinued punishment frequency seems to lose its differential control of behavior. Thus, intermittent punishment may suppress behavior less than continuous punishment or frequent punishment only when some reward is available.

\section{REFERENCES}

AZRIN, N. H., HOLZ, W. C., \& HAKE, D. F. Fixed-ratio punishment. Journal of the Experimental Analysis of Behavior, 1963, 6, 141-148. BEECROFT, R. S., \& KRUGER, B. M. VR 2 interresponse times. Psychonomic Science, 1967, 9, 383-384.

CHURCH, R. M., \& RAYMOND, G. A. Influence of the schedule of positive reinforcement on punished behavior. Journal of Comparative \& Physiological Psychology, 1967, 63, 329-332.

HOLZ, W. C. Punishment and rate of positive reinforcement. Journal of the Experimental Analysis of Behavior, 1968, 11, 285-293. NOTE

1. This research was supported by Grant MH 11734-02 from the National Institutes of Health to Judson S. Brown. 